\title{
Quantum Entanglement of Moving Bodies
}

\author{
Robert M. Gingrich and Christoph Adami \\ Quantum Computing Technologies Group, Jet Propulsion Laboratory 126-347 \\ California Institute of Technology, Pasadena, CA 91109-8099
}

(February 1, 2008)

\begin{abstract}
We study the properties of quantum information and quantum entanglement in moving frames, and show that the entanglement between the spins and the momenta of two particles become mixed when viewed by a moving observer. A pair of particles that is entangled in spin but not momentum in one reference frame, may, in another frame, be entangled in momentum at the expense of spinentanglement. Similarly, entanglement between momenta may be transferred to spin under a Lorentz transformation. While spin and momentum entanglement each is not Lorentz invariant, the joint entanglement of the wave function is.
\end{abstract}

PACS numbers: 03.30+p,03.67-a,03.65.Ud

Mostly, theories in physics are born out of necessity, but not always. The thermodynamics of moving bodies for example (relativistic thermodynamics) was a hotly contested topic without resolution [1-4] (but see [5]) mostly because no experiment required it. As a sideeffect, it was learned that the temperature concept in relativistic thermodynamics is ambiguous simply because radiation that is perfectly black-body in an inertial frame is not thermal if viewed from a moving frame $[6,5]$. This is an interesting result for information theory [7], however, since if probability distributions can depend on the inertial frame, then so can Shannon entropy and information. Even more interesting are the consequences for quantum information theory, where quantum entanglement plays the role of the primary resource in quantum computation and communication [8]. Relativistic quantum information theory may become a necessary theory in the near future, with possible applications to quantum teleportation [9] entanglement-enhanced communication [10], quantum clock synchronization, and quantum-enhanced global positioning [11].

Entanglement is a property unique to quantum systems. Two systems (microscopic particles or even macroscopic bodies [12]) are said to be quantum entangled if they are described by a joint wave function that cannot be written as a product of wave functions of each of the subsystems (or, for mixed states, if a density matrix cannot be written as a weighted sum of product density matrices). The subsystems can be said not to have a state of their own, even though they may be arbitrarily far apart. The entanglement produces correlations between the subsystems that go beyond what is classically possible [13]. It is this feature that enables quantum communication protocols such as teleportation and super-dense coding. However, the preparation, sharing, and purification of entanglement is usually a complicated and expensive process that requires great care. It is therefore of some importance to understand all those processes that might affect quantum entanglement (in particular those processes that lead to decoherence). It was shown recently that Lorentz boosts can affect the marginal entropy of a single quantum spin [14]. Here, we determine that the entanglement between two systems depends on the frame in which this entanglement is measured. We show that a fully entangled spin- $1 / 2$ system (a Bell state) loses entanglement if observed by a Lorentz-boosted observer. Thus, Lorentz boosts introduce a transfer of entanglement between degrees of freedom, that could be used for entanglement manipulation. While the entanglement between spin or momentum alone may change due to Lorentz boosts, the entanglement of the entire wave function (spin and momentum) is invariant.

In order to define the momentum eigenstates for a massive particle with spin, we start by defining the rest frame eigenstates

$$
\begin{aligned}
P^{\mu}|\mathbf{0} \lambda\rangle & =|\mathbf{0} \lambda\rangle p_{0}^{\mu}, \\
\mathbf{J}^{2}|\mathbf{0} \lambda\rangle & =|\mathbf{0} \lambda\rangle s(s+1), \\
J_{z}|\mathbf{0} \lambda\rangle & =|\mathbf{0} \lambda\rangle \lambda,
\end{aligned}
$$

where $p_{0}^{\mu}=(m, \mathbf{0}), s$ is the total angular momentum of the particle, and $\lambda$ is the $z$ component of angular momentum. Since the particle is at rest, $s$ and $\lambda$ are the spin and the $z$ component of the spin for the particle respectively.

We define a momentum state by acting on the rest frame state with a pure Lorentz boost

$$
|\mathbf{p} \lambda\rangle \equiv L\left(\xi_{\mathbf{p}}\right)|\mathbf{0} \lambda\rangle .
$$

where $L\left(\xi_{\mathbf{p}}\right)$ is a boost such that

$$
L\left(\xi_{\mathbf{p}}\right)(m, \mathbf{0})=\left(\sqrt{\mathbf{p}^{2}+m^{2}}, \mathbf{p}\right)
$$

where the rapidity, $\xi_{\mathbf{p}}$, is given by

$$
\begin{aligned}
\sinh \left|\xi_{\mathbf{p}}\right| & =\frac{|\mathbf{p}|}{m} \\
\frac{\xi_{\mathbf{p}}}{\left|\xi_{\mathbf{p}}\right|} & =\frac{\mathbf{p}}{|\mathbf{p}|} .
\end{aligned}
$$


In what follows, we use $\mathbf{p}$ to represent the 4-vector $\left(\sqrt{\mathbf{p}^{2}+m^{2}}, \mathbf{p}\right)$ unless it is ambiguous.

The effect of an arbitrary Lorentz transformation $\Lambda$ (rotation and boost) on a momentum eigenstate is

$$
\begin{aligned}
\Lambda|\mathbf{p} \lambda\rangle & =\Lambda L\left(\xi_{\mathbf{p}}\right)|\mathbf{0} \lambda\rangle \\
& =L\left(\xi_{\Lambda \mathbf{p}}\right) L\left(\xi_{\Lambda \mathbf{p}}\right)^{-1} \Lambda L\left(\xi_{\mathbf{p}}\right)|\mathbf{0} \lambda\rangle .
\end{aligned}
$$

Since $L\left(\xi_{\Lambda \mathbf{p}}\right)^{-1} \Lambda L\left(\xi_{\mathbf{p}}\right)$ leaves $\mathbf{0}$ invariant it must be a rotation. These rotations are called the Wigner rotations $R(\Lambda, \mathbf{p})$, and they act only on the rest frame spin component $\lambda$. Hence, we can write

$$
\Lambda|\mathbf{p} \lambda\rangle=\sum_{\lambda^{\prime}}\left|\Lambda \mathbf{p} \lambda^{\prime}\right\rangle D_{\lambda^{\prime}, \lambda}^{(s)}(R(\Lambda, \mathbf{p}))
$$

where $D_{\lambda^{\prime}, \lambda}^{(s)}(R)$ is the spin $s$ representation of the rotation $R$. Here, we restrict ourselves to $s=1 / 2$, but the generalization to larger spins is straightforward. For a review of momentum eigenstates and spin, see [15]. Since a local unitary transformation will not affect any measure of entanglement $[18,19]$, the unitary transformation $\Lambda$ on the infinite dimensional space of momentum and rest frame spin will not change the entanglement between two particles provided we do not trace out a part of the wavefunction. However, in looking at the entanglement between spins, tracing out over the momentum is implied.

The wavefunction for two massive spin- $1 / 2$ particles can be written as

$$
\left|\Psi_{A A^{\prime} B B^{\prime}}\right\rangle=\iint \sum_{\lambda \sigma} g_{\lambda \sigma}(\mathbf{p}, \mathbf{q})|\mathbf{p} \lambda\rangle_{A A^{\prime}}|\mathbf{q} \sigma\rangle_{B B^{\prime}} \tilde{\mathrm{d}} \mathbf{p} \widetilde{\mathrm{d}} \mathbf{q},
$$

where $\widetilde{d} \mathbf{p}$ and $\widetilde{d} \mathbf{q}$ are the Lorentz-invariant momentum integration measures given by

$$
\widetilde{\mathrm{d}} \mathbf{p} \equiv \frac{\mathrm{d}^{3} \mathbf{p}}{2 \sqrt{\mathbf{p}^{2}+m^{2}}}
$$

and the functions $g_{\lambda \sigma}(\mathbf{p}, \mathbf{q})$ must satisfy

$$
\sum_{\lambda \sigma} \iint\left|g_{\lambda \sigma}(\mathbf{p}, \mathbf{q})\right|^{2} \widetilde{\mathrm{d}} \mathbf{p} \widetilde{\mathrm{d}} \mathbf{q}=1
$$

To an observer in a frame Lorentz transformed by $\Lambda^{-1}$ the state $\left|\Psi_{A A^{\prime} B B^{\prime}}\right\rangle$ appears to be transformed by $\Lambda \otimes \Lambda$. Using Eq. (10), and a change of variables for $\mathbf{p}, \mathbf{q}, \lambda$ and $\sigma, g_{\lambda \sigma}(\mathbf{p}, \mathbf{q})$ goes through the following transformation

$$
g_{\lambda \sigma}(\mathbf{p}, \mathbf{q}) \rightarrow \sum_{\lambda^{\prime} \sigma^{\prime}} U_{\lambda, \lambda^{\prime}}^{\left(\Lambda^{-1} \mathbf{p}\right)} U_{\sigma, \sigma^{\prime}}^{\left(\Lambda^{-1} \mathbf{q}\right)} g_{\lambda^{\prime} \sigma^{\prime}}\left(\Lambda^{-1} \mathbf{p}, \Lambda^{-1} \mathbf{q}\right)
$$

where we defined

$$
U_{\lambda, \lambda^{\prime}}^{(\mathbf{p})} \equiv D_{\lambda, \lambda^{\prime}}^{\left(\frac{1}{2}\right)}(R(\Lambda, \mathbf{p}))
$$

for compactness of notation. The Lorentz transformation can be viewed as a unitary operation, $R(\Lambda, \mathbf{p})$, conditioned on $\mathbf{p}$ acting on the spin, followed by a boost $\mathbf{p} \rightarrow \Lambda \mathbf{p}$ on the momentum represented by the circuit diagram in fig. 1.

By writing $\left|\Psi_{A A^{\prime} B B^{\prime}}\right\rangle$ as a density matrix and tracing over the momentum degrees of freedom, the entanglement between $A$ and $B$, (that is, between the spin-degrees of freedom) can be obtained by calculating Wootters's concurrence $[16,17]$

$$
C\left(\rho_{A B}\right)=\max \left\{\lambda_{1}-\lambda_{2}-\lambda_{3}-\lambda_{4}, 0\right\},
$$

where $\left\{\lambda_{1}, \lambda_{2}, \lambda_{3}, \lambda_{4}\right\}$ are the square roots of the eigenvalues of the matrix $\rho_{A B} \tilde{\rho}_{A B}$, and $\tilde{\rho}_{A B}$ is the "timereversed" matrix [16]

$$
\tilde{\rho}_{A B}=\left(\sigma_{y} \otimes \sigma_{y}\right) \rho_{A B}^{\star}\left(\sigma_{y} \otimes \sigma_{y}\right)
$$

The first step in calculating the Lorentz transformed concurrence is to find an explicit form for $U_{\lambda, \lambda^{\prime}}^{(\mathbf{p})}$. Since any Lorentz transformation $\Lambda$ can be written as a rotation $R(\boldsymbol{\Theta})$ followed by a boost $L(\xi)$ (see also Eq. (8)) it is clear that for a pure rotation, $U_{\lambda, \lambda^{\prime}}^{(\mathbf{p})}$ does not depend on p. Hence, tracing over the momentum after a rotation will not change the concurrence. Therefore, we can look only at pure boosts, and without loss of generality we may choose boosts in the $z$-direction. Writing the momentum 4-vector in polar coordinates as

$$
\mathbf{p}=[E, p \cos (\theta) \sin (\phi), p \sin (\theta) \sin (\phi), p \cos (\phi)],
$$

we obtain

$$
U^{(\mathbf{p})}=\left[\begin{array}{cc}
\alpha & \beta e^{-i \theta} \\
-\beta e^{i \theta} & \alpha
\end{array}\right]
$$

where

$$
\begin{aligned}
& \alpha=\sqrt{\frac{E+m}{E^{\prime}+m}}\left[\cosh \left(\frac{\xi}{2}\right)+\frac{p \cos (\phi)}{(E+m)} \sinh \left(\frac{\xi}{2}\right)\right], \\
& \beta=\frac{p \sin (\phi)}{\sqrt{(E+m)\left(E^{\prime}+m\right)}} \sinh \left(\frac{\xi}{2}\right),
\end{aligned}
$$

and

$$
E^{\prime}=E \cosh (\xi)+p \cos (\phi) \sinh (\xi) .
$$

Here we use $\xi=|\xi|$ as the rapidity of the boost in the $z$ direction.

For momentum distributions in this letter we use a "relativistic gaussian" with width $\sigma_{r}$

$$
f(\mathbf{p})=\sqrt{\frac{1}{N\left(\sigma_{r}\right)} \exp -\left(\frac{p^{2}}{2 \sigma_{r}^{2}}\right)},
$$

which differs from the standard gaussian only in the normalization $N\left(\sigma_{r}\right)$, chosen in accordance with (12). 
For a spin Bell state $\left|\phi^{+}\right\rangle$with momenta in a product gaussian, we have

$$
g_{\lambda \sigma}(\mathbf{p}, \mathbf{q})=\frac{1}{\sqrt{2}} \delta_{\lambda \sigma} f(\mathbf{p}) f(\mathbf{q}) .
$$

Boosting this state, we move some of the spin entanglement to the momentum. Tracing out the momentum from the Lorentz-transformed density matrix destroys some of the entanglement, and hence the concurrence in the moving frame diminishes. The change in concurrence depends only on the ratio $\sigma_{r} / m$ and $\xi$. Figure 2 shows the concurrence vs. rapidity $\xi$, for $\sigma_{r} / m=1$ and 4. The decrease from the maximum value (the concurrence is one for Bell states) documents the boost-induced decoherence of the spin entanglement.

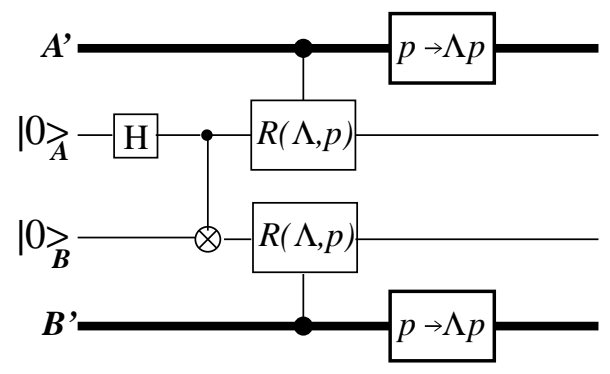

FIG. 1. Circuit diagram for a Lorentz boost on a state with spins in a $\left|\phi^{+}\right\rangle$state. Lines representing momentum degrees of freedom are bold.

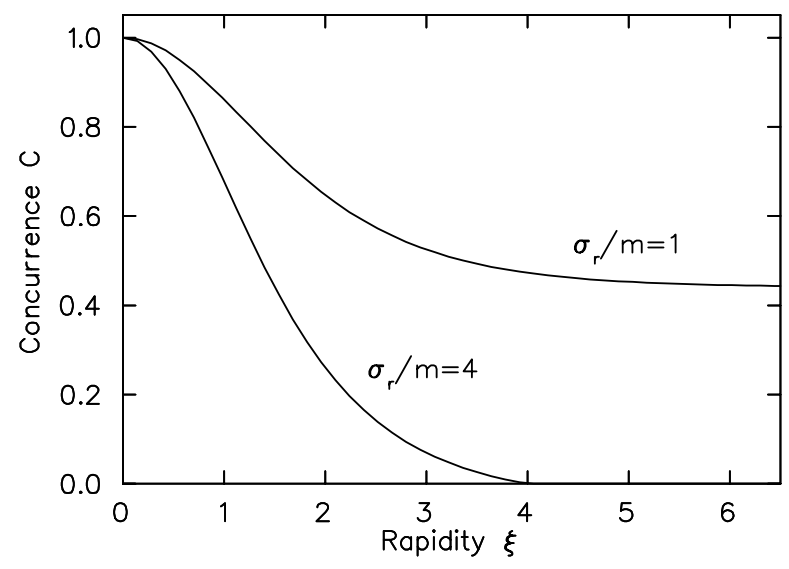

FIG. 2. Spin-concurrence as a function of rapidity, for an initial Bell state with momenta in a product gaussian. Data is shown for $\sigma_{r} / m=1$ and $\sigma_{r} / m=4$.

In the limit $\xi \rightarrow \infty$ (boost to the speed of light), the concurrence saturates, i.e., it reaches a constant value that depends on the mass of the particles and the shape of the momentum distribution. In this particular example, it depends on the ratio $\sigma_{r} / m$. The saturation level decreases as $\sigma_{r} / m$ increases until $\sigma_{r} / m \simeq 3.377$ when the saturation level becomes zero. Note that in the limit of "pure" momentum states (plane waves), the spins undergo local unitary rotations but entanglement transfer does not occur, as was observed in $[20,21]$. The reason for the saturation can be seen by examining (19) in the limit $\xi \rightarrow \infty$

$$
\begin{aligned}
\lim _{\xi \rightarrow \infty} \alpha & =\sqrt{\frac{E+m}{2(E+p \cos (\phi))}}\left(1+\frac{p \cos (\phi)}{E+m}\right) \\
\lim _{\xi \rightarrow \infty} \beta & =\frac{p \sin (\phi)}{\sqrt{2(E+m)(E+p \cos (\phi))}} .
\end{aligned}
$$

The parameter $\beta$ represents the amount of rotation due to the boost. If we maximize $\beta$ with respect to $\phi$ we obtain

$$
\beta \rightarrow \frac{\frac{p}{m}}{1+\sqrt{1+\left(\frac{p}{m}\right)^{2}}},
$$

which is a monotonically increasing function of $p / m$. For a particle of mass $m$ and magnitude of momentum $p$, Eq. (27) represents the maximal amount of rotation due to a boost. By increasing $\sigma_{r} / m$ in our example, we effectivly increase this limit for $\beta$, and hence how much we can alter the concurrence. Note that since for large $p / m$ the rotation $\beta$ tends to one, the Lorentz transformation is equivalent to a conditional spin flip in this regime.

If boosts can disentangle spins, can they transfer entanglement from the momentum degrees of freedom to an unentangled spin wavefunction? Indeed this is possible. One way to achieve this is to take any of the resulting states after boosting the state in Eq. (24) and apply the inverse boost to increase concurrence to one. Note that the increase in spin entanglement comes at the expense of a loss of momentum entanglement, since the entanglement between all degrees of freedom (spin and momentum) is constant under Lorentz transformations.

Simply reversing a previously applied Lorentz transformation as in the last example is not a very satisfying way to create entanglement. Is there a way we could create an unentangled state in the laboratory frame that would appear entangled to a moving observer? Consider the state

$$
\left|\Psi_{A A^{\prime} B B^{\prime}}\right\rangle=\frac{1}{\sqrt{2}}\left(|\mathbf{p},-\mathbf{p}\rangle\left|\phi^{+}\right\rangle+\left|\mathbf{p}_{\perp},-\mathbf{p}_{\perp}\right\rangle\left|\phi^{-}\right\rangle\right)
$$

where $\mathbf{p}$ and $\mathbf{p}_{\perp}$ are both in the $x, y$ plane, have the same magnitude $p$, and are perpendicular. We could imagine such a state arising from a particle decay where the products are restricted to movement in the $x$ or $y$ axes with a conditional $\sigma_{z}$ gate on the perpendicular direction. The reduced density matrix $\rho_{A B}$ for this wavefunction is separable, and its concurrence vanishes. However, taking the large $p$ limit in Eqs. (25) and (26) and choosing $\phi$ and $\theta$ appropriate for $\mathbf{p}$ and $\mathbf{p}_{\perp}$ in the $x$ and $y$ directions respectively, one can show that for a large boost in the $z$ direction both $\left|\phi^{+}\right\rangle$and $\left|\phi^{-}\right\rangle$are transformed into the 
$\left|\psi^{-}\right\rangle$state and hence the spins are maximally entangled in this reference frame. In fact, the concurrence as a function of $\mathbf{p}$ and $\xi$ is given by

$$
C\left(\rho_{A B}\right)=\frac{p^{2}\left(\cosh ^{2}(\xi)-1\right)}{\left(\sqrt{1+p^{2}} \cosh (\xi)+1\right)^{2}},
$$

when choosing $m=1$. Note that the concurrence is greater than zero whenever $p$ and $\xi$ are nonzero, and as $p$ and $\xi$ become large the concurrence tends to one. So, if we restrict ourselves to spin measurements, an observer in the rest frame of the decay particle cannot use entanglement as a resource (e.g., for teleportation, superdense coding, etc.) while the moving observer can. Such a purification of spin entanglement is not always possible, however, and the following theorem characterizes the limitations:

Theorem: The entanglement between the spin and momentum parts of a pure state wave function, $\left|\Psi_{A A^{\prime} B B^{\prime}}\right\rangle$, must be non-zero to allow the spin entanglement to increase under Lorentz transformations.

Proving the contrapositive, starting with a product state of the form

$$
\left|\Psi_{A A^{\prime} B B^{\prime}}\right\rangle=|\psi\rangle_{A^{\prime} B^{\prime}}|\phi\rangle_{A B}
$$

and applying boosts of the form $\Lambda \otimes \Lambda$ or even $\Lambda \otimes \Lambda^{\prime}$, we obtain

$$
\rho_{A B}=\sum_{i} p_{i} U_{A}^{i} \otimes V_{B}^{i}|\phi\rangle\langle\phi| U_{A}^{i \dagger} \otimes V_{B}^{i \dagger},
$$

where $U_{A}^{i}$ and $V_{B}^{i}$ are unitary operators and the sum $\sum_{i} p_{i}$ will be an integral for certain states $|\psi\rangle$. We can now plug Eq. (31)into any entanglement monotone $E(\rho)$ and obtain the inequality

$$
\begin{aligned}
E\left(\rho_{A B}\right) & \leq \sum_{i} p_{i} E\left(U_{A}^{i} \otimes V_{B}^{i}|\phi\rangle\right) \\
& =\sum_{i} p_{i} E(|\phi\rangle) \\
& =E(|\phi\rangle)
\end{aligned}
$$

where inequality (32) comes from the definition of an entanglement monotone [22]. Hence, the spin entanglement can only decrease after a Lorentz transformation.

Note that this theorem does not hold if arbitrary unitary operations are applied to a particle's spin and momentum degrees of freedom (for instance a swap gate), but it does hold for the entire class of unitaries realized by Lorentz transformations.

We have investigated the properties of moving entangled pairs of massive particles. Because Lorentz boosts entangle the spin and momentum degrees of freedom, entanglement can be transferred between them. This is true for single particles [14], and we have shown here that it is true for pairs, where the Lorentz boost affects the entanglement between spins. Quite generally, we can say that fully entangled spin states will (depending on the initial momentum wavefunction) most likely decohere due to the mixing with momentum degrees of freedom. We also note, however, that such mixing can purify spin entanglement if the momentum degrees are entangled with the spin. The physics of creating entanglement between spins and between momenta is very different. Thus, the possibility of entanglement transfer via Lorentz boosts could conceivably, in special situations, lead to simplified state preparation and purification protocols.

We would like to thank Jonathan Dowling, and the members of the JPL Quantum Computing Group, for useful discussions and encouragement. We also acknowledge Daniel Terno for valuable suggestions. This work was carried out at the Jet Propulsion Laboratory (California Institute of Technology) under a contract with the National Aeronautics and Space Administration, with support from the National Security Agency, the Advanced Research and Development Activity, the Defense Advanced Research Projects Agency, the National Reconnaissance Office, and the Office of Naval Research.

[1] A. Einstein, Über das Relativitätsprinzip und die aus demselben gezogenen Folgerungen, Jahrb. f. Rad. und Elekt. 4, 411 (1907); M. Planck, Zur Dynamik bewegter Systeme, Ann. d. Phys. 26, 1 (1908);

[2] H. Ott, Lorentz-Transformation der Wärme, Z. f. Physik 175, 70 (1963); H. Arzeliès, Transformation relativiste de la température et de quelques autres grandeurs thermodynamiques. Nuov. Cim. 35,792 (1964).

[3] R Aldrovandi and J. Gariel, On the riddle of the moving thermometer. Phys. Lett. A 170, 5 (1992).

[4] K.P. Trout and A.J. Greiner, Special relativistic temperature transformation, Nuov. Cim. 113B, 1439 (1998).

[5] P.T. Landsberg and G.E.A. Matsas, Laying the ghost of the relativistic temperature transformation. Phys. Lett. A223, 401 (1996).

[6] P.J.B. Peebles and D.T. Wilkinson. Comment on the anisotropy of the primeval fireball. Phys. Rev. 174, 2168 (1968).

[7] C.E. Shannon, A mathematical theory of communication, Bell Syst. Tech. J. 27, 379 (1948); ibid, 623 (1948).

[8] M.A. Nielsen and I.L. Chuang. Quantum Computation and Quantum Communication (Cambridge University Press, 2000).

[9] C.H. Bennett et al., Teleporting an unknown quantum state via dual classical and Einstein-Podolsky-Rosen channels, Phys. Rev. Lett. 70, 1895(1993).

[10] C.H. Bennett and S.J. Wiesner, Communication via oneand two-particle operators on Einstein-Podolsky-Rosen states, Phys. Rev. Lett. 69, 2881 (1992).

[11] R. Josza, D.S. Abrams, J.P. Dowling, and C.P. Williams. Quantum atomic clock synchronization based on shared 
prior entanglement. Phys. Rev. Lett. 85, 2010 (2000); V. Giovanetti, S. Lloyd, and L. Maccone, Quantumenhanced positioning and clock synchronization. Nature 412, 417 (2001).

[12] B. Julsgaard, A. Kozhekin, and E.S. Polzik. Experimental long-lived entanglement of two macroscopic objects. Nature 413, 400 (2001).

[13] N.J. Cerf and C. Adami, Negative entropy and information in quantum mechanics. Phys. Rev. Lett. 79, 5194 (1997).

[14] A. Peres, P.F. Scudo, and D.R. Terno, Quantum entropy and special relativity. Phys. Rev. Lett. 88, 230402 (2002).

[15] W. Tung. Group Theory in Physics (World Scientific Publishing, 1985)

[16] W.K. Wootters, Entanglement of formation of an arbitrary state of two qubits. Phys. Rev. Lett. 80, 2245
(1998).

[17] Because after the Lorentz transform the spins are in a mixed state, entanglement measures based on pure states cannot be used.

[18] G. Vidal, Entanglement Monotones. J. Mod. Opt. 47, 355 (2000).

[19] S. Parker and S. Bose and M.B. Plenio, Entanglement Quantification and Purification in Continuous-Variable Systems. Phys. Rev. A 61, 032305 (2000)

[20] M. Czachor, Einstein-Podolsky-Rosen-Bohm experiment with massive particles. Phys. Rev. A 55, 72 (1997)

[21] P.A. Alsing and G.J. Milburn, Lorentz invariance of entanglement. Quantum Information and Computation (to be published).

[22] G. Vidal, Entanglement monotones. J. Mod. Opt. 47355 (2000) 